\title{
Synthesis of rotaxane cross-linked polymers derived from vinyl monomers using a metal-containing supramolecular cross-linker
}

\author{
Masahiro Ogawa ${ }^{1}$, Hiromitsu Sogawa ${ }^{1}$, Yasuhito Koyama ${ }^{1}$ and Toshikazu Takata ${ }^{1,2}$ \\ Polymer Journal (2015) 47, 580-584; doi:10.1038/pj.2015.34; published online 17 June 2015
}

\section{INTRODUCTION}

Rotaxane cross-linked polymers $(\mathrm{RCPs})^{1-3}$ are a type of network polymer $^{4-7}$ with topological cross-linking points. Several studies have been performed regarding the synthesis of RCPs using several types of macrocycles such as cyclodextrins ${ }^{8-11}$ and crown ethers. ${ }^{12-14}$ RCPs possess unique properties including swelling, elasticity and stressreleasing ability, which have also been investigated. Compared with chemical gels (CGs), RCPs prominently feature a movable cross-linker at the cross-linking points. A considerable number of studies have been conducted, primarily on the properties of slide-ring gel, involving poly(ethylene glycol) chains penetrating the cavity of the cyclodextrin moieties. Slide-ring gel exhibits a high elasticity arising from the restoring force of the entropy elasticity of the interpenetrating network. ${ }^{9,10}$ Although numerous attempts have been made to study the properties of RCPs, the mechanisms underlying their specific properties are still controversial because the synthesis of RCPs is hindered by the ring size, the polarity inside the cavity, the properties of the polymer backbone and a number of other issues.

The metal-templated threading method is currently being investigated for use in high-yield RCP synthesis because of its ability to form interlocked complexes. ${ }^{15-17}$ Previously, we reported a successful approach for RCP synthesis via vinyl polymerization using a 4-vinylpyridine (VP)-coordinated bis-macrocyclic metal-containing supramolecular cross-linker $\left(\mathrm{MSC}_{\mathrm{VP}}\right) .{ }^{18}$ However, the main chains of RCPs are limited to poly(4-vinylpyridine), and the utility of the MSC has not been sufficiently studied.

Here, we synthesized RCPs from several types of vinyl polymers, including commercial vinyl monomers such as styrene, methyl methacrylate (MMA), $N$-isopropylacrylamide (NIPAM) and $n$-butyl acrylate (BA) in place of VP (Figure 1). The swelling and physical properties of the RCPs as well as the steric effects of the bulky vinyl monomers introduced into the RCPs were examined in comparison to the CGs synthesized using a commercial chemical cross-linker.

\section{MATERIALS AND METHODS \\ Materials}

The acetonitrile-coordinated metal-containing supramolecular cross-linker $\left(\mathrm{MSC}_{\mathrm{AC}}\right)$ and 4-(tris(4-(tert-butyl)phenyl)methyl)phenol were prepared following previously described methods. ${ }^{18,19}$ All other materials were commercially available and used without further purification.

\section{Measurement}

${ }^{1} \mathrm{H}$ and ${ }^{13} \mathrm{C}$ nuclear magnetic resonance (NMR) spectra were recorded on a JEOL AL-400 spectrometer (JEOL, Tokyo, Japan) using $\mathrm{CDCl}_{3}$ and dimethyl sulfoxide (DMSO- $d_{6}$ ) as solvents. Residual non-deuterated solvent or tetramethylsilane was used as an internal standard. Glass transition temperatures $\left(T_{\mathrm{g}}\right)$ were measured using a Shimadzu DSC-60 instrument (Shimadzu, Kyoto, Japan) under a nitrogen atmosphere (heating rate of $10^{\circ} \mathrm{C} \mathrm{min}^{-1}$ and nitrogen flow rate of $50 \mathrm{ml} \mathrm{min}^{-1}$ ).

Thermogravimetric analyses were performed using a Shimadzu TGA-50 (Kyoto, Japan) instrument in a nitrogen atmosphere (heating rate of $10^{\circ}$ $\mathrm{C} \mathrm{min}^{-1}$ and nitrogen flow rate of $50 \mathrm{ml} \mathrm{min}^{-1}$ ). Fourier transform infrared spectra were recorded on a Jasco FT/IR-460 Plus spectrophotometer (Tokyo, Japan). Melting points were measured using a Stuart Scientific SMP3 melting point apparatus (Bibby Scientific, Stone, UK). Matrix-assisted laser desorption/ ionization time of flight mass spectrometry spectra were measured with a Shimadzu AXIMA-CFR mass spectrometer using a dithranol matrix. The fast atom bombardment high-resolution mass spectra (FAB-HRMS; JMS-700) and inductively coupled plasma mass spectrometry (Agilent 77003) spectra were requested and obtained from the National University Corporation Tokyo Institute of Technology Center for Advanced Materials Analysis.

\section{Compound TBPMA}

4-(Tris(4-(tert-butyl)phenyl)methyl)phenol (TBPMA; $300 \mathrm{mg}, 0.59 \mathrm{mmol}$ ) and 2-isocyanatoethyl methacrylate (karenz MOI, Showa Denko (Tokyo, Japan), $190 \mathrm{mg}, 1.2 \mathrm{mmol}$ ) were dissolved in $\mathrm{CH}_{2} \mathrm{Cl}_{2}(9.0 \mathrm{ml})$. Dibutyltin dilaurate $(10 \mu \mathrm{l})$ was added as a catalyst at room temperature, and the solution was stirred for $3 \mathrm{~h}$. The reaction mixture was concentrated in vacuo. The crude product was purified by column chromatography on a silica gel $\left(\mathrm{CH}_{2} \mathrm{Cl}_{2}\right.$ : hexane $=1: 1)$ to yield TBPMA $(300 \mathrm{mg}, 0.45 \mathrm{mmol}, 77 \%)$ as a white solid with the following characteristics: $\mathrm{mp} 205.0-206.0^{\circ} \mathrm{C} ;{ }^{1} \mathrm{H} \mathrm{NMR}\left(400 \mathrm{MHz}, \mathrm{CDCl}_{3}\right.$,

${ }^{1}$ Department of Organic and Polymeric Materials, Tokyo Institute of Technology, Tokyo, Japan and ${ }^{2}$ Advanced Catalytic Transformation for Carbon Utilization (ACT-C), JST, Tokyo, Japan

Correspondence: Professor T Takata, Department of Organic and Polymeric Materials, Tokyo Institute of Technology, 2-12-1 (H-126), Ookayama, Meguro, Tokyo 152-8552, Japan. 
$298 \mathrm{~K}): \delta 7.23$ (d, $J=8.4 \mathrm{~Hz}, 6 \mathrm{H}), 7.18(\mathrm{~d}, J=8.4 \mathrm{~Hz}, 2 \mathrm{H}), 7.09(\mathrm{~d}, J=8.4 \mathrm{~Hz}$, $6 \mathrm{H}), 6.99(\mathrm{~d}, J=8.4 \mathrm{~Hz}, 2 \mathrm{H}), 6.15(\mathrm{~s}, 1 \mathrm{H}), 5.62(\mathrm{~s}, 1 \mathrm{H}), 4.30(\mathrm{t}, J=5.6 \mathrm{~Hz}, 2 \mathrm{H})$, 3.59 (dd, $J=5.6,6.0 \mathrm{~Hz}, 2 \mathrm{H}), 1.97$ (s, 3H), 1.30 (s, 27H) p.p.m.; ${ }^{13} \mathrm{C} \mathrm{NMR}$
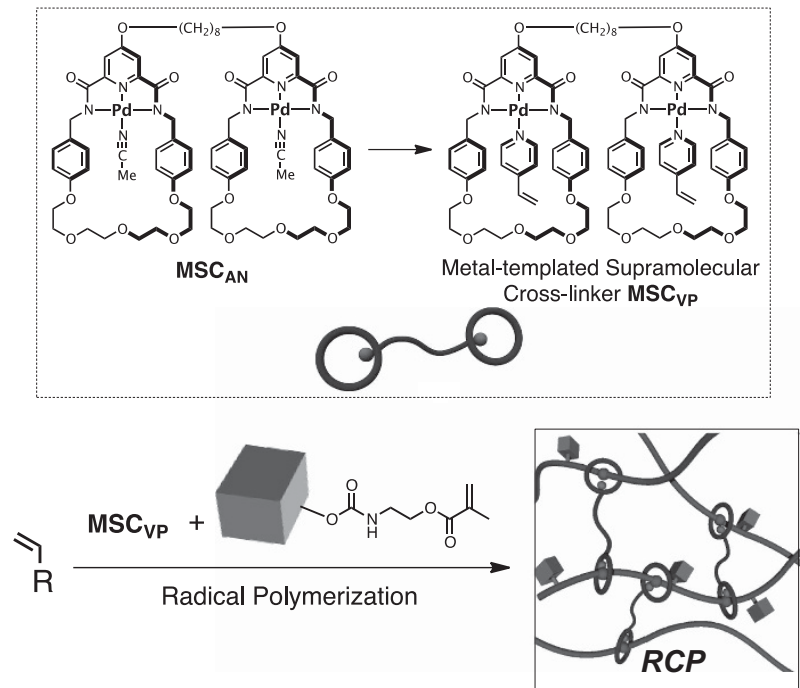

Figure 1 The chemical structure of the bifunctional Pd-containing macrocycles, acetonitrile-coordinated metal-containing supramolecular crosslinker and 4-vinylpyridine-coordinated bis-macrocyclic metal-containing supramolecular cross-linker $\left(\mathrm{MSC}_{\mathrm{VP}}\right.$ ) and the strategy for synthesis of the rotaxane cross-linked polymers via radical polymerization of the monomers in the presence of a bulky vinyl monomer and $\mathrm{MSC}_{\mathrm{VP}}$. A full color version of this figure is available at Polymer Journal online. $\left(100 \mathrm{MHz}, \mathrm{CDCl}_{3}, 298 \mathrm{~K}\right): \delta 154.6,148.6,148.3,144.3,143.7,135.8,132.0$, 130.6, 126.1, 124.0, 119.9, 63.3, 63.2, 40.3, 34.2, 31.3, 18.2 p.p.m.; IR (KBr) $\nu$ 3406, 3386, 3087, 3034, 2961, 2904, 2868, 1749, 1729, 1638, 1535, 1498, 1458, 1399, 1363, 1321, 1298, 1268, 1217, 1173, 1108, 1062, 1018, 944, 842, 824, 760, 705, $633 \mathrm{~cm}^{-1}$; FAB-HRMS (matrix: 3-nitrobenzyl alcohol (NBA)) calculated for $\mathrm{C}_{44} \mathrm{H}_{53} \mathrm{NNaO}_{4}\left[\mathrm{M}+\mathrm{H}^{+}\right]$: 660.4053, found: 660.4067 .

\section{Typical procedure for the preparation of RCP}

A solution of $\mathrm{MSC}_{\mathrm{AN}}(22 \mathrm{mg}, 14 \mu \mathrm{mol})$ and VP $(3.0 \mathrm{mg}, 2.8 \mu \mathrm{mol})$ in DMF $(0.40 \mathrm{ml})$ was stirred for $2 \mathrm{~h}$ at room temperature to afford $\mathrm{MSC}_{\mathrm{VP}}$ in situ. To the solution, TBPMA (180 mg, $0.28 \mathrm{mmol}$ ) and the vinyl monomer (styrene: $300 \mathrm{mg}$, MMA: $140 \mathrm{mg}$, NIPAM: $160 \mathrm{mg}$, BA: $360 \mathrm{mg}, 2.8 \mathrm{mmol}$ ) were added, and the mixture was degassed by repeated freeze-thaw cycling. 2,2'-azobis (isobutyronitrile) (AIBN) $(5.1 \mathrm{mg}, 30 \mu \mathrm{mol})$ was then added to the mixture, warmed to $60^{\circ} \mathrm{C}$ and stirred for $16 \mathrm{~h}$ to obtain a gel. The obtained gel was swollen in $\mathrm{CHCl}_{3}(10 \mathrm{ml})$ overnight to remove impurities and dried under vacuum to give the corresponding RCP $\left(\mathrm{RCP}_{\text {St-en10 }}: 490 \mathrm{mg}, 96 \%\right.$, $\mathrm{RCP}_{\text {MMA-en10: }} 440 \mathrm{mg}, 92 \%, \mathrm{RCP}_{\mathrm{NIPAM}-\mathrm{en} 10}: 590 \mathrm{mg}, 89 \%$ and $\mathrm{RCP}_{\mathrm{BA}-\mathrm{en} 10}$ : $500 \mathrm{mg}, 98 \%)$ as a reddish gel.

\section{Typical procedure for the preparation of CG}

TBPMA (2.0 mol\%: $37 \mathrm{mg}, 56 \mu \mathrm{mol}$ and $10 \mathrm{~mol} \%: 180 \mathrm{mg}, 0.28 \mathrm{mmol}$ ) and BA (360 mg, $2.8 \mathrm{mmol}$ ) were added to a solution of diethylene glycol dimethacrylate $(3.4 \mathrm{mg}, 14 \mu \mathrm{mol})$ and VP $(3.0 \mathrm{mg}, 2.8 \mu \mathrm{mol})$ in DMF $(0.4 \mathrm{ml})$ by degassing by repeated freeze-thaw cycling. AIBN $(1.8 \mathrm{mg}, 14 \mu \mathrm{mol})$ was then added to the mixture, warmed to $60^{\circ} \mathrm{C}$, and stirred for $16 \mathrm{~h}$ to obtain a gel, which was swollen in $\mathrm{CHCl}_{3}(10 \mathrm{ml})$ overnight to remove the impurities and then dried under vacuum to give the corresponding CG (CG $\mathrm{CG}_{\mathrm{BA}-\mathrm{en} 2}: 403 \mathrm{mg}, 100 \%$ and $\mathrm{CG}_{\mathrm{BA}-\mathrm{en10}}$ : $530 \mathrm{mg}, 97 \%$ ) as a reddish gel.

a

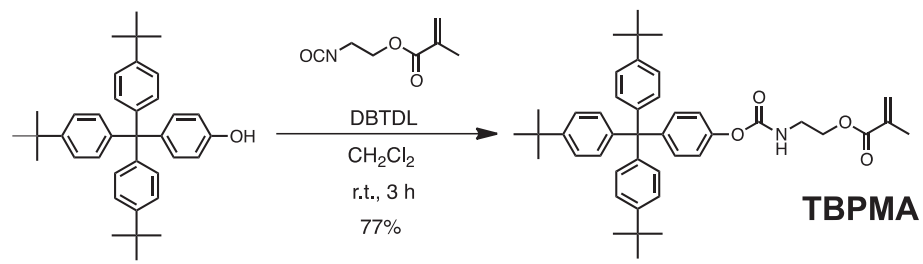

b
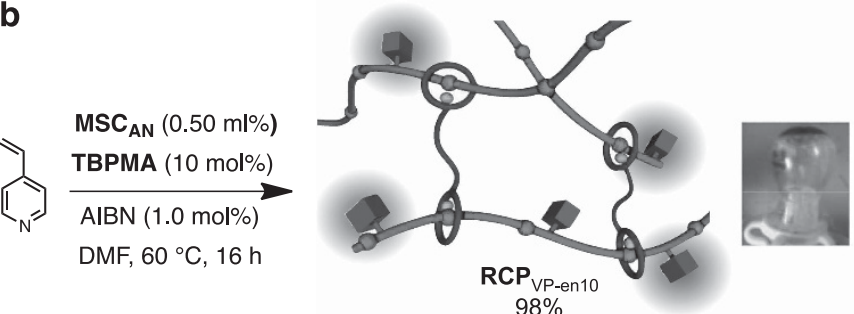

Addition of DMF DMF, $60^{\circ} \mathrm{C}, 16 \mathrm{~h}$

$98 \%$

C
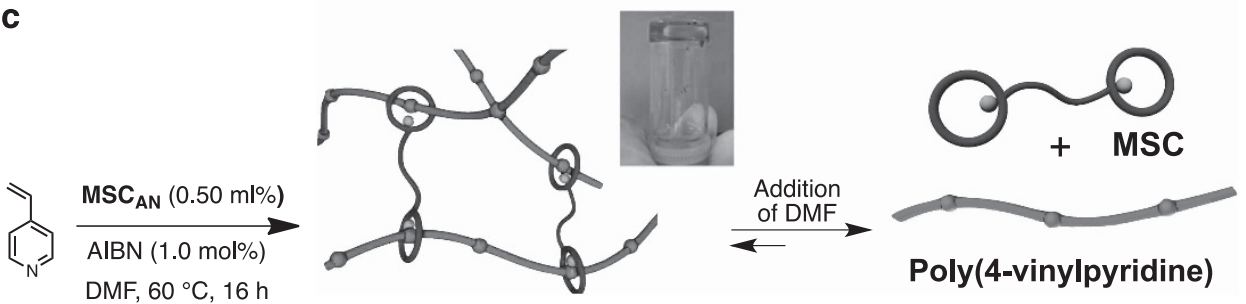

PseudoRCP

Scheme 1 (a) Synthesis of 4-(tris(4-(tert-butyl)phenyl)methyl)phenol (TBPMA). (b) Synthesis of non-deslipping RCPVP-en10 obtained using 10 mol\% of TBPMA. (c) Pseudorotaxane cross-linked polymer prepared without TBPMA obtained without using TBPMA. A full color version of this figure is available at Polymer Journal online. 


\section{RESULTS AND DISCUSSION}

\section{Synthesis of the bulky vinyl monomer (TBPMA)}

In our previous work, RCPs with an end-cap moiety originating from a 4-tert-butylstyrene comonomer were constructed to prevent the deslippage of wheel components. ${ }^{18}$ However, the bulkiness of the tert-butyl phenyl component was not sufficient for the $\mathrm{MSC}_{\mathrm{VP}}$. We therefore developed a novel end-capping reagent, TBPMA, to obtain more stable RCPs from various vinyl monomers. TBPMA was synthesized in three steps from 4-tert-butylbenzoate, and its structure was fully characterized using ${ }^{1} \mathrm{H}$ NMR, ${ }^{13} \mathrm{C}$ NMR and Fourier transform infrared spectroscopy as shown in Scheme 1a and Supplementary Scheme S1. A low-mode sampling calculation of TBPMA was used to determine its effectiveness as a stopper. The size of the monomer TBPMA was calculated to be $11.6 \AA$ (Supplementary Figure S1), whereas that of the macrocycle of the cross-linker was $5.96 \AA .{ }^{16}$ This clearly shows that TBPMA is sterically large enough to prevent the deslippage of bis-macrocyclic compounds from their polymer skeletons.

\section{Synthesis of rotaxane cross-linked polymers containing several vinyl polymers}

Next, a vinylpyridine monomer, TBPMA (10 mol\%) and $\mathrm{MSC}_{\mathrm{AN}}$ ( $0.5 \mathrm{~mol} \%)$, were radically polymerized, using AIBN as an initiator, for $16 \mathrm{~h}$ to yield a red gel product, $\mathrm{RCP}_{\mathrm{VP}-\mathrm{en} 10}$, as illustrated in Scheme 1b. $\mathrm{RCP}_{\mathrm{VP}-\mathrm{en10}}$ was insoluble in all solvents, but could be swollen with solvents such as $\mathrm{CHCl}_{3}, \mathrm{DMF}, \mathrm{MeOH}$ and THF. Its swellability will be discussed later. The pseudorotaxane cross-linked polymer prepared without TBPMA (pseudoRCP $\mathrm{VP}_{\mathrm{VP}}$ ), as in the previous report, was completely dissolved under dilute conditions that prompted dissociation of the $\mathrm{MSC}_{\mathrm{AN}}$ from the trunk polymer chain (Scheme 1c). ${ }^{18}$ This clearly shows that the formation of a stable interlocked structure was maintained without deslippage by introducing TBPMA.

In the interest of contributing to the development of new materials, the synthesis of RCPs with various vinyl monomers was carried out using an acetonitrile-anchoring bis-macrocyclic compound, $\mathrm{MSC}_{\mathrm{AN}}$ and TBPMA. First, a styrene monomer was radically polymerized in place of VP. However, instead of the formation of an RCP, polystyrene (PSt) was obtained. This is because of the absence of a pyridine moiety that is essential for coordination with the Pd bis-macrocycle. To overcome this issue, coordination of $\mathrm{MSC}_{\mathrm{AN}}$ and $\mathrm{VP}$ (ratio 1:1) was performed to yield a VP-anchoring bis-macrocyclic metal-containing supramolecular cross-linker, $\mathrm{MSC}_{\mathrm{VP}}$, as shown in Figure 2a. This product readily copolymerized with other types of vinyl monomers to obtain several RCPs. The polymerization of styrene was carried out for $16 \mathrm{~h}$ using $0.5 \mathrm{~mol} \%$ of $\mathrm{MSC}_{\mathrm{VP}}$ and $10 \mathrm{~mol} \%$ of TBPMA to obtain a rotaxane gel cross-linked polymer, $\mathrm{RCP}_{\text {St-en10, }}$ as shown in Figure $2 \mathrm{~b}$. Moreover, MMA and BA, which exhibit low glass transition temperatures $\left(T_{\mathrm{g}}\right)$, were used to obtain $\mathrm{RCP}_{\mathrm{MMA} \text {-en10 }}$ and rubbery $\mathrm{RCP}_{\mathrm{BA}-\mathrm{en} 10}$ with a high yield following the same protocol for the synthesis of

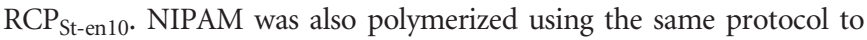
obtain $\mathrm{RCP}_{\mathrm{NIPAM}-e n 10}$ with an excellent yield.

\section{Swelling and thermal properties of RCPs}

It is expected that the obtained RCPs will maintain the specific characteristics of the primary monomers even though $0.5 \mathrm{~mol} \%$ of pyridine moieties and $10 \mathrm{~mol} \%$ of a bulky stopper were copolymerized in this system. Here, we focus on the swelling ratio of RCPs in various solvents, as demonstrated in Figure 2c.

Firstly, RCPs exhibited high swelling properties in $\mathrm{CHCl}_{3}$, and moderate swelling in DMF irrespective of the monomer structure. A high swelling ratio in $\mathrm{CHCl}_{3}$ may be related to the presence of the 4-(tris
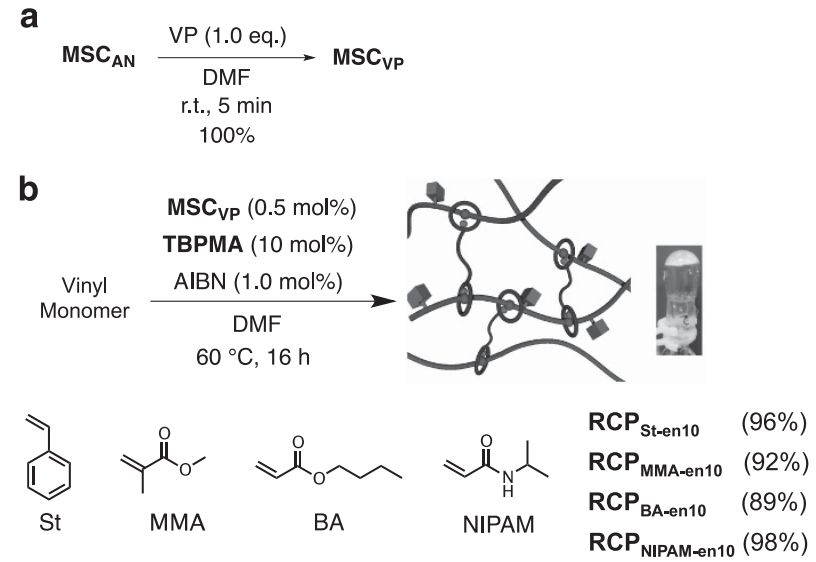

c

\begin{tabular}{|c|c|c|c|c|c|c|c|}
\hline \multirow{2}{*}{ Entry } & \multirow{2}{*}{ Monomer } & \multirow{2}{*}{$R C P$} & \multicolumn{3}{|c|}{ Swelling ratio $(\%)$} & \multirow{2}{*}{$T_{g}\left({ }^{\circ} \mathrm{C}\right)$} & \multirow{2}{*}{$T_{d 5}\left({ }^{\circ} \mathrm{C}\right)$} \\
\hline & & & $\mathrm{MeOH}$ & $D M F$ & $\mathrm{CHCl}_{3}$ & & \\
\hline 1 & St & $\mathrm{RCP}_{\text {St-en10 }}$ & 30 & 430 & 600 & 63.0 & 157 \\
\hline 2 & MMA & $\mathrm{RCP}_{\text {MMA-en10 }}$ & 40 & 680 & 1400 & 82.0 & 198 \\
\hline 3 & BA & $\mathrm{RCP}_{\mathrm{BA} \text {-en10 }}$ & 40 & 110 & 1500 & -47.0 & 315 \\
\hline 4 & NIPAM & $\mathrm{RCP}_{\text {NPAM-en10 }}$ & 180 & 660 & 2000 & 91.4 & 185 \\
\hline
\end{tabular}

${ }^{a}$ Swelling ratio $[\%]=\left\{\left(\mathrm{W}_{\text {swollen }}-\mathrm{W}_{\text {dry }}\right) / \mathrm{W}_{\text {dry }}\right\} \times 100$

Figure 2 (a) Synthesis of the vinylpyridine-containing bis-macrocyclic compound, $\mathrm{MSC}_{\mathrm{VP}}$. (b) The polymerization protocols for the rotaxane crosslinked polymers with several monomers including styrene, methyl methacrylate, $N$-isopropylacrylamide and $n$-butyl acrylate yielding $\mathrm{RCP}_{\text {St-en10, }}$ RCP ${ }_{\text {MMA-en 10, }} \mathrm{RCP}_{\text {NIPAM-en10 }}$ and $\mathrm{RCP}_{\mathrm{BA} \text {-en10, respectively. (c) The swelling }}$ ratio and the thermal properties of RCPSt-en10, RCPMM A-en10, RCPNIPAM-en 10 and RCPBA-en10. A full color version of this figure is available at Polymer Journal online.

(4-(tert-butyl)phenyl)methyl)phenyl group that is compatible with the $\mathrm{CHCl}_{3}$ solvent. In the case of $\mathrm{RCP}_{\mathrm{NIPAM}-e n 10}$, the swelling ratio in $\mathrm{MeOH}$ was higher than that of the other RCPs because the NIPAM monomer is highly polar. However, $\mathrm{RCP}_{\mathrm{St}-\mathrm{en} 10}$ and $\mathrm{RCP}_{\mathrm{BA}-\mathrm{en} 10 \text {, which }}$ contain substituents with relatively low polarities in the backbone chain, displayed low swelling properties in DMF compared with $\mathrm{RCP}_{\text {MMA-en10 }}$ and $\mathrm{RCP}_{\mathrm{NIPAM} \text {-en10. }}$. To evaluate the thermal properties of the dry RCPs, we carried out differential scanning calorimetry and thermogravimetric analysis measurements. All RCPs exhibited $T_{\mathrm{g}}$ values that are almost identical to those of the homopolymers obtained from the corresponding monomers of $100,105,120$ and $-54^{\circ} \mathrm{C}$ for PSt, PMMA, PNIPAM and $\mathrm{PBA}$, respectively. ${ }^{20}$ As expected, the rubber-like $\mathrm{RCP}_{\mathrm{BA}-\mathrm{en} 10}$ showed the lowest $T_{\mathrm{g}}$ compared with the other RCPs. Moreover, the obtained RCPs showed moderate thermal stability $\left(T_{\mathrm{d} 5} \geqslant 157^{\circ} \mathrm{C}\right)$. These results indicate that the properties of moderately stable RCPs derived from various vinyl monomers can be adjusted using our method without losing the original physical characteristics of the monomers. Presumably, the removal of the palladium metal from the RCPs enhances the mobility of the polymer chains. Although we attempted to remove the palladium metal by treating with hydrochloric acid, the Pd was only partially desorbed (Supplementary Material).

\section{RCP versus CG}

To clarify the effect of the movable cross-linking points on the RCPs, a CG was prepared using a chemical cross-linker, CC (diethylene glycol dimethacrylate), to introduce covalently cross-linked points into the 
Table 1 Preparation of RCP and CG with BA in the presence of TBPMA

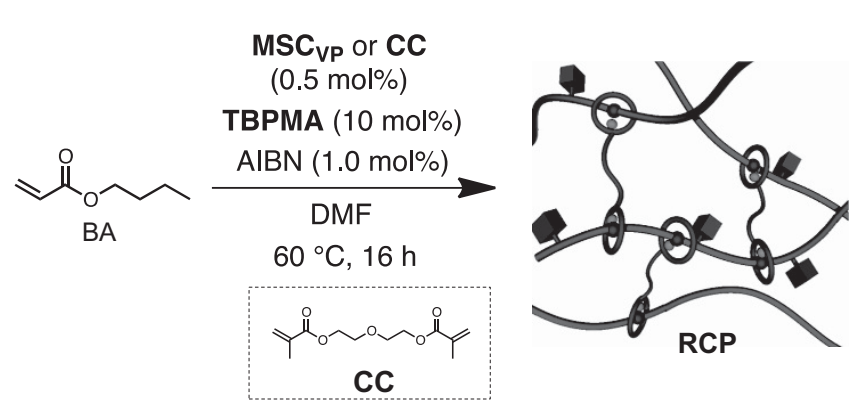

Swelling ratio ${ }^{a}(\%)$

\begin{tabular}{llclccc} 
Entry & Cross-linker & TBPMA (mol \%) & Yield (\%) & $\mathrm{MeOH}$ & $\mathrm{DMF}^{2}$ & $\mathrm{CHCl}_{3}$ \\
\hline 1 & MSC $_{\mathrm{VP}}$ & 10 & RCP $_{\text {BA-en10 }}(89)$ & 40 & 110 & 1500 \\
2 & MSC $_{\mathrm{VP}}$ & 2 & RCP $_{\text {BA-en2 }}(95)$ & 40 & 670 & 1900 \\
3 & CC & 10 & CG $_{\text {BA-en10 }}(97)$ & 50 & 1100 & 4900 \\
4 & CC & 2 & CG $_{\text {BA-en2 }}(100)$ & 60 & 1400 & 4400 \\
\hline
\end{tabular}

Abbreviations: CC, chemical cross-linker; CG, chemical gel; BA, n-butyl acrylate; MSC $\mathrm{vP}$, 4-vinylpyridine-coordinated bis-macrocyclic metal-containing supramolecular cross-linker; TBPMA, 4-(tris(4-(tert-butyl)phenyl)methyl)pheno

aswelling ratio $(\%)=\left\{\left(W_{\text {swollen }}-W_{\text {dry }}\right) / W_{\text {dry }}\right\} \times 100$

A full color version of this table is available at Polymer Journal online.

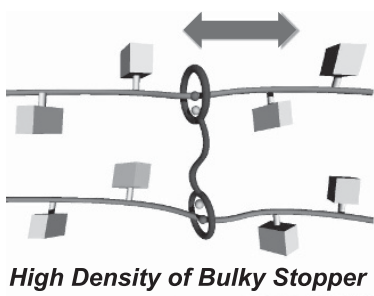

Low Swelling Property

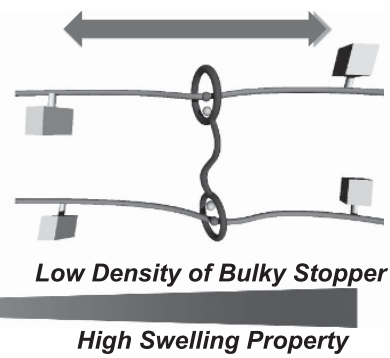

High Swelling Property
Figure 3 Schematic illustration of the mobile rotaxane cross-link points with different bulky end-cap (4-(tris(4-(tert-butyl)phenyl)methyl)phenol) (TBPMA) content. A full color version of this figure is available at Polymer Journal online.

network. $\mathrm{CG}_{\mathrm{BA}-\mathrm{en} 10}$ and $\mathrm{RCP}_{\mathrm{BA}-\text { en10 }}$ were obtained using $10 \mathrm{~mol} \%$ of TBPMA with the same cross-linker ratio $(0.5 \mathrm{~mol} \%)$, whereas $\mathrm{CG}_{\mathrm{BA}-\mathrm{en} 2}$ and $\mathrm{RCP}_{\mathrm{BA} \text {-en2 }}$ were obtained using $2.0 \mathrm{~mol} \%$ of TBPMA (Table 1). $\mathrm{CG}_{\mathrm{BA}-\mathrm{en} 10}$ and $\mathrm{CG}_{\mathrm{BA}-\mathrm{en} 2}$, which were obtained using same amount of cross-linker, showed nearly identical swelling behavior in $\mathrm{MeOH}, \mathrm{DMF}$ and $\mathrm{CHCl}_{3}$. The similarity in the swelling behavior of these CGs indicates that the amount of TBPMA does not affect the swelling behavior of formed gels. However, $\mathrm{RCP}_{\mathrm{BA}-\mathrm{en} 2}$ exhibited increased swellability than $\mathrm{RCP}_{\mathrm{BA}-\mathrm{en} 10}$ in all solvents. This was particularly pronounced in DMF. This difference can be attributed to the difference in the TBPMA content between the two systems, with $2.0 \mathrm{~mol} \%$ of the end-cap component in $\mathrm{RCP}_{\mathrm{BA}-\mathrm{en} 2}$ versus $10 \mathrm{~mol} \%$ in $\mathrm{RCP}_{\mathrm{BA}-\mathrm{en10}}$. This suggests that the RCP swellability is strongly dependent on the ratio of the bulky end-cap component, TBPMA. A decrease in the stopper ratio resulted in a wide range of mobility of the bis-macrocycles through the polymer skeleton, leading to high stress relaxation in the movable rotaxane network (Figure 3). This behavior induces the favorable swellability in $\mathrm{RCP}_{\mathrm{BA}-\mathrm{en} 2}$. Although the
CG exhibited a higher swelling ratio than the RCP, the RCP had a higher cross-link density than that of the CG when applied to the modified Flory-Rehner equation (Supplementary Material). This difference may be attributed to the higher reactivity of the VP monomer of $\mathrm{MSC}_{\mathrm{VP}}$ than that of the methacrylate moieties of $\mathrm{CC}$, although several possible reasons are conceivable. We have not yet obtained the appropriate RCP films for tensile tests, although polymerizations were carried out with various radical initiators under many different conditions.

\section{CONCLUSION}

We describe an efficient protocol for the synthesis of RCPs with various polymer skeletons including PSt, PNIPAM, PMMA and PBA. It is noteworthy that the metal-containing supramolecular crosslinker, $\mathrm{MSC}_{\mathrm{VP}}$, a vinylpyridine-coordinated bis-macrocycle, performs as a vinyl cross-linking agent for radical polymerization. Furthermore, the use of only $0.5 \mathrm{~mol} \%$ of the rotaxane cross-linker is enough to obtain a movable cross-linked gel. The obtained RCPs possess physical properties similar to the corresponding polymer skeletons. This report is the first example of a macrocyclic effect in which a low amount of a bulky stopper gives rise to an RCP with higher swellability, due to the higher mobility of the wheel through the polymer chains. Further investigation of this new RCP synthesis strategy using other polymerization methods such as cationic, anionic and condensation polymerization are now in progress.

\section{CONFLICT OF INTEREST}

The authors declare no conflict of interest.

\section{ACKNOWLEDGEMENTS}

This work was financially supported by the ACT-C program from JST, a Grantin-Aid for Scientific Research from the Ministry of Education, Culture, Sports, Science and Technology, Japan (No. 24685023).

1 Takata, T. Polyrotaxane and polyrotaxane network: supramolecular architectures based on the concept of dynamic covalent bond chemistry. Polym. J. 38, 1-20 (2006).

2 Ito, K. Slide-ring materials using topological supramolecular architecture. Curr. Opin. Solid State Mater. Sci. 14, 28-34 (2010).

3 Koyama, Y. Synthesis of topologically crosslinked polymers with rotaxanecrosslinking points. Polym. J. 46, 315-322 (2014).

4 Ogoshi, T., Ichihara, Y., Yamagishi, T. \& Nakamoto, Y. Supramolecular polymer networks from hybrid between graphene oxide and per-6-amino- $\beta$-cyclodextrin. Chem. Commun. 46, 6087-6089 (2010).

5 Suzaki, Y., Taira, T. \& Osakada, K. Physical gels based on supramolecular gelators, including host-guest complexes and pseudorotaxanes. J. Mater. Chem. 21, 930-938 (2011).

6 Zhang, M., Xu, D., Yan, X., Chen, J., Dong, S., Zheng, B. \& Huang, F. Self-healing supramolecular gels formed by crown ether based host-guest interactions. Angew. Chem. Int. Ed. 51, 7011-7015 (2012)

7 McKee, J. R., Appel, E. A., Seitsonen, J., Kontturi, E., Scherman, O. A. \& Ikkala, O. Healable, stable and stiff hydrogels: combining conflicting properties using dynamic and selective three-component recognition with reinforcing cellulose nanorods. Adv. Funct. Mater. 24, 2706-2713 (2014).

8 Okumura, Y. \& Ito, K. The polyrotaxane gel: a topological gel by figure-of-eight crosslinks. Adv. Mater. 13, 485-487 (2001).

9 Mayumi, K., Tezuka, M., Bando, A. \& Ito, K. Mechanics of slide-ring gels: novel entropic elasticity of a topological network formed by ring and string. Soft. Matter $\mathbf{8}$, 8179-8183 (2012).

10 Kato, K., Yasuda, T. \& Ito, K. Viscoelastic properties of slide-ring gels reflecting sliding dynamics of partial chains and entropy of ring components. Macromolecules 46, 310-316 (2013)

11 Arai, T., Jang, K., Koyama, Y., Asai, S. \& Takata, T. Versatile supramolecular crosslinker: a rotaxane cross-linker that directly endows vinyl polymers with movable crosslinks. Chem. Eur. J. 19, 5917-5923 (2013).

12 Oku, T., Furusho, Y. \& Takata, T. A concept for recyclable cross-linked polymers: topologically networked polyrotaxane capable of undergoing reversible assembly and disassembly. Angew. Chem. Int. Ed. 43, 966-969 (2004). 
13 Kohsaka, Y., Nakazono, K., Koyama, Y. \& Takata, T. Size-complementary rotaxane crosslink: effect on stabilization and degradation of supramolecular network. Angew. Chem. Int. Ed. 50, 4872-4875 (2011).

14 lijima, K., Kohsaka, Y., Koyama, Y., Nakazono, K., Uchida, S., Asai, S. \& Takata, T. Stimuli-degradable cross-linked polymers synthesized by radical polymerization using a size-complementary [3]rotaxane cross-linker. Polym. J. 46, 67-72 (2013).

15 Beves, J. E., Blight, B. A., Campbell, C. J., Leigh, D. A. \& McBurney, R. T. Strategies and tactics for the metal-directed synthesis of rotaxanes, knots, catenanes and higher order links. Angew. Chem. Int. Ed. 50, 9260-9327 (2011).

16 Furusho, Y., Matsuyama, T., Takata, T., Moriuchi, T. \& Hirao, T. Synthesis of nove interlocked systems utilizing a palladium complex with 2,6-pyridinedicarboxamidebased tridentate macrocyclic ligand. Tetrahedron Lett. 45, 9593-9597 (2004).
17 Miyagawa, N., Watanabe, M., Matsuyama, T., Koyama, Y., Moriuchi, T., Hirao, T. Furusho, Y. \& Takata, T. Successive catalytic reactions specific to Pd-based rotaxane complexes as a result of wheel translation along the axle. Chem. Commun. 46, 1920-1922 (2010)

18 Ogawa, M., Kawasaki, A., Koyama, Y. \& Takata, T. Synthesis and properties of polyrotaxane network prepared from Pd-templated bis-macrocycle as a topological cross-linker. Polym. J. 43, 909-915 (2011).

19 Gibson, H. W., Lee, S., Engen, P. T., Lecavalier, P., Sze, J., Shen, Y. X. \& Bheda, M. New triarylmethyl derivatives: "Blocking Groups" for rotaxanes. J. Org. Chem. $\mathbf{5 8}$ 3748-3756 (1993).

20 Brandrup, J., Immergut, E. H. \& Grulke, E. A. Polymer Handbook (Wiley-Interscience, Weinheim, Germany, 1999).

Supplementary Information accompanies the paper on Polymer Journal website (http://www.nature.com/pj) 